\title{
Classification of Images in Fog and Fog-Free Scenes for Use in Vehicles
}

\author{
Mario Pavlić ${ }^{1}$, Gerhard Rigoll ${ }^{2}$ and Slobodan Ilić $^{3}$
}

\begin{abstract}
Today modern vehicles are often equipped with a camera, which captures the scene in front of the vehicle. The recognition of weather conditions with this camera can help to improve many applications as well as establish new ones. In this article we will show how it is possible to distinguish between scenes with clear and foggy weather situations. The proposed method uses only gray-scale images as input signal and is running in real time. Using spectral features and a simple linear classifier, we can achieve high detection rates in both daytime and night-time scenes. Furthermore, we will show that in our application area these features outperform others.
\end{abstract}

\section{INTRODUCTION}

Camera based driver assistance systems are increasingly used in current vehicles. Well known applications are for example the Lane Departure Warning, the Speed Limit Information, the High Beam Assistant or the Adaptive Cruise Control. All these applications have in common that they recognize specific objects in the image like lanes, traffic signs, lights or vehicles. However, recognizing general image properties, such as current weather conditions, has so far been given little attention. In this work we are focusing on the problem of detecting fog, i.e. distinguishing between clear and foggy weather situations on the basis of camera images. On the one hand, this information can help to improve existing applications, i.e. by restoring foggy images using known methods to improve object detection or by adjusting the strategy of the High Beam Assistant, and on the other hand establish new application areas like the Local Hazard Warning of poor visibility or ambient sensitive rear lights. For the classification of images into fog and fog-free, we use a single grayscale camera, which is mounted in the vehicle behind the rearview mirror. But first, it is important to clarify the term fog. According to the international definition in meteorology fog is defined as a cloud that touches the ground and causes a visibility range of less than 1,000 $\mathrm{m}$ [1]. The visibility range thereby describes the longest distance at which a black object of adequate size can be observed towards the horizon. The International Commission on Illumination (CIE) recommends a threshold of $5 \%$ [2]. To concretize the term fog in the application area of individual road traffic following categorization has been proposed [3]:

- No Fog: Visibility range $>1,000 \mathrm{~m}$.

\footnotetext{
${ }^{1}$ M. Pavlić is with BMW Group, Traffic Technology and Traffic Management, 80788 Munich, Germany, Mario.Pavlic@bmw. de

${ }^{2}$ G. Rigoll is with Department of Human-Machine-Communication, Technische Universität München, Theresienstr. 90, 80333 Munich, Germany, Rigolletum.de

${ }^{3} \mathrm{~S}$. Ilić is with Department of Informatics, Technische Universität München, Boltzmannstr. 3, 85748 Garching, Germany, Slobodan. Ilic@in.tum.de
}

- Low Fog: Visibility range between $300-1,000 \mathrm{~m}$.

- Fog: Visibility range between $100-300 \mathrm{~m}$.

- Dense Fog: Visibility range $<100 \mathrm{~m}$.

Depending on the target application it is to decide when is referred to fog. For the Local Hazard Warning for example this is only the category Dense Fog, for the High Beam Assistant this may be the categories Fog and Dense Fog.

In the next section we will look at related work in the field of image based fog detection and visibility range estimation. Then we will present our approach to classify images into fog and fog-free scenes and in the end we finally show how well our method in both daytime and night-time scenes work.

\section{RELATED WORK}

Estimating the visibility range in fog was first investigated by Bush and Debers for the stationary use [4]. They used a camera mounted on a traffic management system. After manually defined a fixed region of interest, the road region, they performed a wavelet based contrast measurement. The image line above which no contrast higher than $5 \%$ appears, defines the visibility range, which could be transformed to world coordinates because of the known camera setup. The first visibility range estimation in a vehicle was introduced by Pomerleau [5]. He used arbitrary road features to determine a percentage visibility range. Further work by Hautière et al. [6], [7], [8] and later by Bronte et al. [9] examined the transition between the road and the sky region to estimate a metric visibility range. While all of these works have been developed for use in daytime scenes, Gallen et al. introduced an approach for night-time scenes [10]. They divided the problem into two scenarios. If no external light sources are present, like streetlights or lights of driving ahead or oncoming vehicles, then the light propagation of the vehicle headlight has been investigated. Therefore from the current driving scene a fixed region of the road has been compared to a template, which was previously recorded in a fog chamber. However, if external light sources were present then their corona was analyzed using a multi-threshold approach.

While all of these approaches try to determine the visibility range at once, we believe that for a robust operation the problem has to be split. First, one has to decide whether at all a fog situation is present. If fog is present, the degree of fog can be quantized by estimating the visibility range $e . g$. by analyzing the contrast along lane markings. The decision whether the vehicle is in a fog situation or not is a scene classification problem, which is well known in literature. Typical challenges are indoor/outdoor scene classification [11], [12], [13], [14], [15], city/landscape scene classification [16], [17], semantic scene classification [18], 
[19] or generally grouping similar images in the field of content-based image retrieval (CBIR) [20]. However, the classification of weather situations for use in the automotive sector has received very little attention. Roser and Moosmann proposed a general method for weather classification based on color and texture features [21]. They determined for the entire image and for twelve subregions histograms of the local contrast, minimum brightness, sharpness, hue and saturation and concatenated them to a large feature vector. The images were then classified as clear weather, light rain or heavy rain by a two-stage linear SVM. They mention that their approach is also applicable to the classification of fog situations, but it wasn't evaluated because of the lack of appropriate images. Furthermore Pavlić et al. [3] proposed a method which is able to distinguish between dense fog and clear weather situations. It is inspired by the work of Oliva and Torralba [18] and uses spectral features which are also known as Gist features.

Our work is an extension of that from Pavlic et al. with an improved detection rate. This is achieved by a modified sampling of the power spectrum with a so called Gabor band pass filter bank and by extracting features from some large overlapping windows in spatial domain. Furthermore we will show, that this approach works also very well for nighttime scenes and that spectral features outperform others in distinguishing between clear and foggy weather situations. Therefore we compare the features used by us with those of Roser and Moosmann and the Wavelet features proposed by Serrano et al. for indoor/outdoor classification [14]. The latter use the coefficients of the Wavelet transform, as used in the JPEG2000 compression standard for image compression, as image descriptors.

\section{METHOD}

As input signal we use grayscale images from a camera available in serial-production vehicles. To model the sensitivity of the human visual system, we first perform a conversion to logarithmic domain [22], [23]

$$
i_{l}(x, y)=\log (i(x, y)+1) .
$$

Afterwards we normalize the image to avoid that some image regions dominate the spectrum and to reduce illumination effects

$$
i^{\prime}(x, y)=\frac{i_{l}(x, y) * h(x, y)}{\varepsilon+\sqrt{\left[i_{l}(x, y) * h(x, y)\right]^{2} * g(x, y)}} .
$$

Thereby $g(x, y)$ describes an isotropic low pass filter with a radial cut off frequency at 0.015 cycles/pixel and $h(x, y)=$ $1-g(x, y)$. The numerator is a high pass filter that cancels the mean intensity of the image. The denominator acts as a local estimator of the variance of the output of the high pass filter. $\varepsilon$ is a constant that avoids noise enhancements in homogeneous image regions. For the normalization we use a square image section and equalize the intensity values to the range of $[0,255]$.
As image features we use the power spectrum

$$
\Gamma\left(f_{x}, f_{y}\right)=\left|I\left(f_{x}, f_{y}\right)\right|^{2},
$$

which is defined as the square magnitude of the Fourier transform for every frequency $f_{x}$ and $f_{y}$ of the image of size $W$ given by

$$
I\left(f_{x}, f_{y}\right)=\sum_{x=0}^{W-1} \sum_{y=0}^{W-1} i^{\prime}(x, y) e^{-j 2 \pi\left(f_{x} x+f_{y} y\right)} .
$$

Before the Fourier transform we furthermore apply a two dimensional Hanning window to avoid leakage effects. As can bee seen in Fig. 1 images with fog present provide a spectrum concentrated around the zero frequency whereas images of clear weather situations have much more high frequency components. This is obvious as fog causes blurring and sharp edges are composed in frequency domain by high and low frequency components whereas weak edges only by low ones.

Next, we perform a two stage feature reduction consisting of sampling the spectrum in the frequency domain and a subsequent feature selection based on Principle Components Analysis (PCA).

The sampling is done by a filter bank of scaled and oriented Gabor filters [24]. The $i$ th Gabor filter is defined as

$$
G_{i}\left(f_{x}, f_{y}\right)=e^{-2 \pi^{2}\left(\sigma_{x}^{2}\left(f_{x}^{\prime}-f_{r}\right)^{2}+\sigma_{y}^{2} f_{y}^{\prime 2}\right)}
$$

with $f_{x}^{\prime}=f_{x} \cos (\theta)+f_{y} \sin (\theta)$ and $f_{y}^{\prime}=-f_{x} \sin (\theta)+$ $f_{y} \cos (\theta)$. Thereby the filters are shifted to the position $f_{r}$ and rotated by $\theta$. So the filters are arranged semicircular around the zero frequency in different frequency bands. An example for a Gabor filter bank is shown in Fig. 2(a). Since we are not necessarily interested in the directional information of frequency components in our application an alternative filter bank configuration is to subsume the Gabor filter of one scale to one so-called Gabor band pass filter as shown in Fig. 2(b).
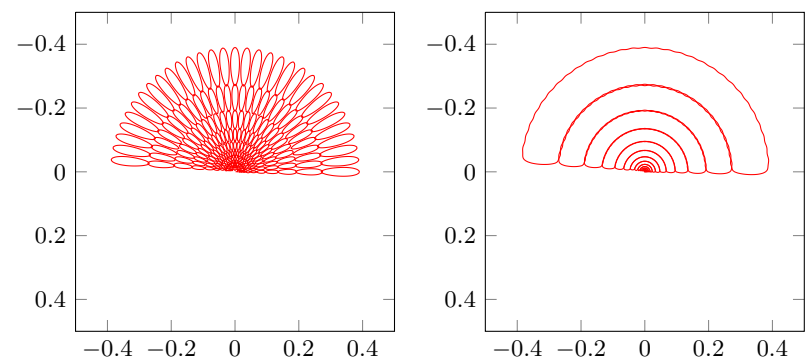

Fig. 2. Examples for Gabor filter banks. The horizontal and vertical axis correspond to the $f_{x}$ and $f_{y}$ frequency, the contours display the $3 \mathrm{~dB}$ cutoff frequency. Left: Gabor filter bank with 16 scales between $0.33 \mathrm{c} / \mathrm{p}$ and $0.002 \mathrm{c} / \mathrm{p}$ with $32,32,28,28,24,24,16,16,12,12,8,8,6,6,4$ and 4 orientations per scale. Right Equivalent Gabor band pass filter bank where the filter of one scale are subsumed to one band pass filter.

So we get a sampled spectrum by the outputs of the filter, the so-called Gabor feature vector

$$
\mathbf{g}=\left\{g_{i}\right\}_{i=1}^{K}
$$



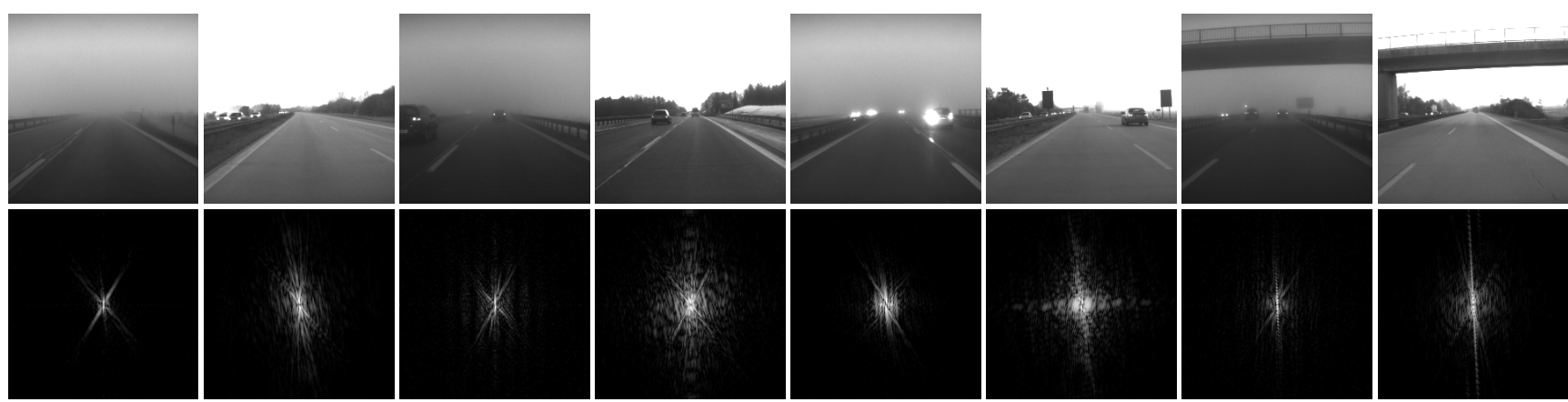

Fig. 1. Images of fog and fog-free scenes and the corresponding power spectra. The power spectra are displayed in logarithmic unit, with zero frequency in the middle and higher frequencies at the border. The images presented above show clear differences in the spectra between similar scenes. While in fog scenes the frequency components are concentrated at the origin, in fog-free scenes they are broadly spread.

where $K$ is the number of filters and

$$
g_{i}=\sum \sum \Gamma\left(f_{x}, f_{y}\right) G_{i}\left(f_{x}, f_{y}\right), i=1,2 \ldots K .
$$

Afterwards we perform a feature selection based on PCA

$$
\boldsymbol{\omega}=\mathbf{W}^{T}(\mathbf{g}-\overline{\mathbf{g}}) .
$$

The mean Gabor feature vector

$$
\overline{\mathbf{g}}=\frac{1}{M} \sum_{m=1}^{M} \mathbf{g}_{m}
$$

and the transformation matrix

$$
\mathbf{W}=\left[\begin{array}{llll}
\mathbf{w}_{1} & \mathbf{w}_{2} & \ldots & \mathbf{w}_{N}
\end{array}\right],
$$

which consists of the first $N$ Eigenvectors of the covariance matrix

$$
\mathbf{C}=\frac{1}{M} \sum_{m=1}^{M}\left(\mathbf{g}_{m}-\overline{\mathbf{g}}\right)\left(\mathbf{g}_{m}-\overline{\mathbf{g}}\right)^{T}
$$

are for this calculated by a set of $M$ training data.

These features are now used for classification. Although Support Vector Machines (SVM) are the state of the art classifiers we use for simplicity a linear classifier based on Fisher's Linear Discriminant Analysis (LDA)

$$
u=\mathbf{d}^{T} \boldsymbol{\omega}=\sum_{n=1}^{N} d_{n} \omega_{n} \begin{cases}>b, & \text { fog } \\ <b, & \text { fog-free }\end{cases}
$$

According to Fisher the weight vector $\mathbf{d}$ is calculated by the covariance matrices $C_{1,2}$ and mean feature vectors $\bar{\omega}_{1,2}$ of the two classes

$$
\mathbf{d}=\left(\mathbf{C}_{1}+\mathbf{C}_{2}\right)^{-1}\left(\overline{\boldsymbol{\omega}}_{1}-\overline{\boldsymbol{\omega}}_{2}\right) .
$$

Because we have chosen the same number of training data for each class the threshold $b$ can be calculated by simply intersecting the probability densities of the decision variable. We assume that $u$ is normal distributed for each class, hence

$$
b=\mathcal{N}_{1}\left(\bar{u}_{1}, \sigma_{1}\right) \cap \mathcal{N}_{2}\left(\bar{u}_{2}, \sigma_{2}\right),
$$

where $\bar{u}_{1,2}$ and $\sigma_{1,2}$ describe the mean and the standard deviation of $u$ for the respective class. This is equivalent to a maximum a posteriori (MAP) estimation. Fig. 3 shows an example output of a training with Fisher LDA.

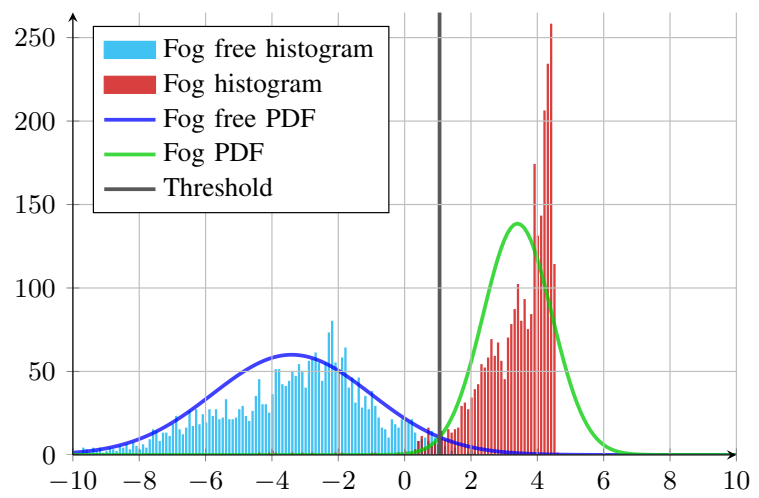

Fig. 3. Result of a exemplary training phase based on the Fisher LDA. In cyan the histogram of fog-free and in red the histogram of fog decision variables is shown. The corresponding normal distributions are shown in blue and green. Note that they are scaled for better visualization. The threshold, resulting from the intersection of the two normal distributions, is shown as a black line. Although the histogram of the fog decision variables does not fit very well with a normal distribution, this approach provides useful results, and also has the advantage of being simple.

\section{A. Configuration for daytime scenes}

For daytime scenes we used the experimentally determined value of $\varepsilon=10$ in the pre-filtering step.

Using the spectrum based on the entire image, $94.35 \%$ of the scenes were accurately classified ${ }^{1}$ with a Gabor band pass filter bank, consisting of 13 frequency bands between 0.33 and $0.005 \mathrm{c} / \mathrm{p}$.

Extracting features from image tiles and combining them in a feature vector is often mentioned to improve the accuracy, in [18] e.g. $8 \times 8$ large overlapping windows were used. In our application this impaired the detection rate. However, using only one line of 8 horizontally aligned windows, in particular the 4th from top, which corresponds to the horizon region in the image, we could improve the accuracy to $95.35 \%$. Thereby we used windows with 64 pixel in size and sampled each one with a Gabor band pass filter bank consisting of 10 frequency bands between 0.33 and $0.014 \mathrm{c} / \mathrm{p}$.

\footnotetext{
${ }^{1}$ The evaluation method will be explained in detail in the next section.
} 

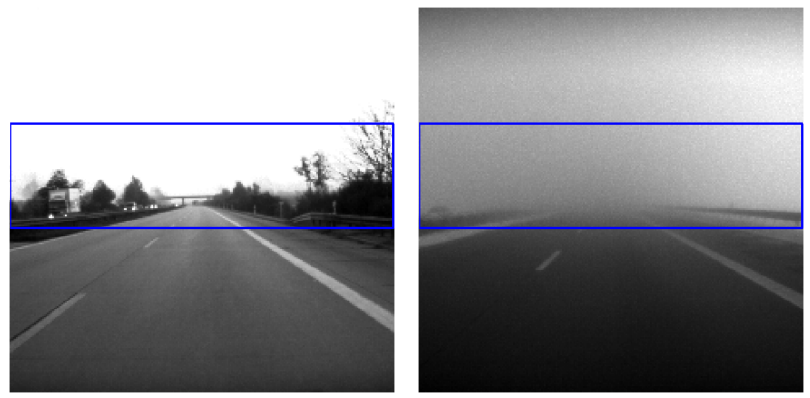

Fig. 4. Used image region to calculate spectral features of eight horizontally large overlapping windows. In the area of the horizon it is most likly to find distant objects, on whose visiblity fog has the most impact, and thus can contribute to the distiction of fog- and fog-free scenes particulaty well.

\section{B. Configuration for night-time scenes}

By switching on the high beams in fog the driver is dazzled by his own light. Therefore it should be possible to distinguish between clear and foggy weather conditions when high beams are switched on. However, our objective was to enable this even without switched high beams, i.e. only with activated low beam. This is much more challenging, since a distinction without the high beam is very difficult even for the driver. To compare the performance of the classification between activated low beam and high beam, for both situations one configurations was determined experimentally.

In the case of night-time scenes and switched low beam, we use a Gabor filter bank consisting of 32 filters, which were positioned in a frequency band at $0.23 \mathrm{c} / \mathrm{p}$. This frequency band corresponds to the second from the outside in Fig. 2(a).

In the case of night-time scenes and switched high beam, we used a Gabor filter bank consisting of 7 frequency bands between 0.33 and $0.04 \mathrm{c} / \mathrm{p}$. It corresponds to the seven outermost frequency bands in Fig. 2(a).

For all night-time scenes we used the experimentally determined value of $\varepsilon=40$ in the pre-filtering step.

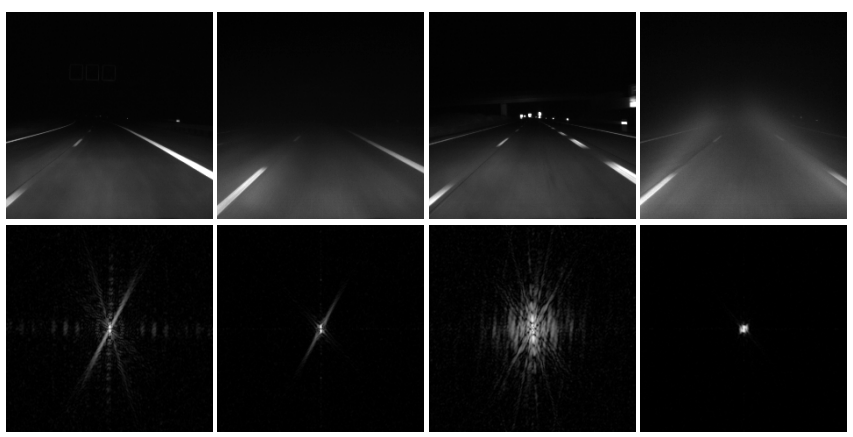

Fig. 5. Examples for clear and fog scenes at night. The top row shows the camera images the bottom row the corresponding power spectra. From left to right: No fog, fog, no fog and high beam, fog and high beam.

\section{EVALUATION}

For evaluation, we used the front camera of a BMW F10, which is mounted behind the rearview mirror and looks in driving direction parallel to the vehicle longitudinal axis. The camera provides grayscale images of resolution $320 \times 240$ pixels at an effective frame rate of $15 \mathrm{fps}$.

Based on this system on German highways images were taken in fog and in clear weather conditions. The daytime scenes were then labeled as category Excluded ${ }^{2}$, No Fog, Low Fog, Fog or Dense Fog. For labeling in each image two horizontal lines were drawn, which under the assumption of a planar road correspond to a distance of $100 \mathrm{~m}$ and $300 \mathrm{~m}$. The images were finally assigned to one category depending on the visibility of the lanes at these lines.

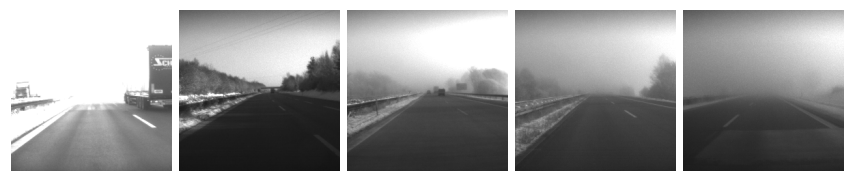

Fig. 6. Example images for labeling categories. Form left to right: Excluded, No Fog, Low Fog, Fog and Dense Fog.

To evaluate the daytime scenes an 8-fold cross-validation was used. For this purpose, eight sample sets were created, each consisting of 5,500 images. Each sample set contains one half fog images and the other half fog-free images. The fog images are from the category Dense Fog, the fogfree images from the categories No Fog and Low Fog. Thus 44,000 images were incorporated into the evaluation of daytime scenes. While compiling the sample sets it was paid attention to group similar types of highway. The A8 highway is e.g. in contrast to A92 very hilly and has a distinct vegetation beside the road. Thus images from the A8 have much higher contrast in clear weather situations. This way we wanted to examine how the system behaves e.g. in basically low-contrast images, if it was trained with basically high-contrast images. In addition to the method presented here, the evaluation was also conducted with the basic approache from Pavlić et al. [3] for daytime scenes, the approach from Roser and Moosmann [21], here called Roser features, and the approach from Serrano et al. [14], here called Wavelet features. When using Roser respectively Wavelet features, the best results were achieved when the features are extracted from the pre-filtered image and then a feature selection was performed by PCA. Moreover the Roser features contain no color information, since only grayscale images were available. Tab. I shows the results of the evaluation of daytime scenes. As can be seen there our method outperforms the others. Besides the highest recognition rate of $95.35 \%$ the lowest standard deviation of the single results of the cross-validation was achieved. This is reflected also in the best result for the worst single result. This shows that our method in comparison to the other is more robust to variations.

Looking at the result of the classification in detail, we can see that our method mainly falsely detected clear weather conditions when despite fog high contrasts appear in the image. This could be observed $e . g$. just before passing bridges or while overtaking trucks. With free road or at a subsequent

\footnotetext{
${ }^{2}$ The category Excluded contains images with overexposure or dazzle.
} 


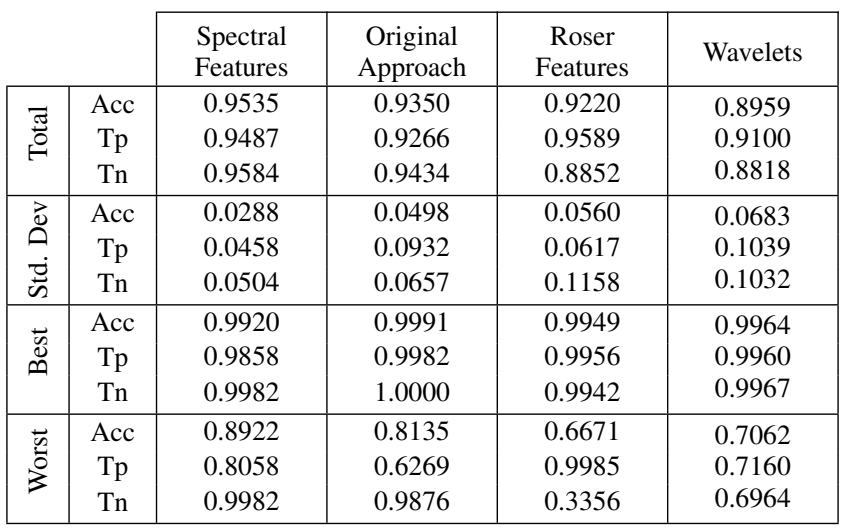

TABLE I: Evaluation results for daytime scenes. The Spectral Features represents the method described here, the Original Approach shows the results from [3], but using a linear classifier based on Fisher LDA. Total accuracy (Acc), true positive rate ( $\mathrm{Tp}$ ) and true negative rate ( $\mathrm{Tn})$ are the mean values of all single results. The true positive rate is the conditional probability that images are classified as fog when labeled as fog, the true negative rate is the conditional probability that images are classified as fogfree when labeled as fog-free. Further important measures are the standard deviations of the single results as well as the best and worst single result regarding the accuracy.

drive rarely occurred false detections. Cases where a fog situation was falsely detected, occurred sporadically, i.e. isolated within a recording, and mostly in low-fog labeled images. Hence, such false detections can be easily fixed by temporal filtering, e.g. by a majority vote of the classification results within a second or through 15 successive frames. Fig. 7 gives some striking classification results for daytime scenes.
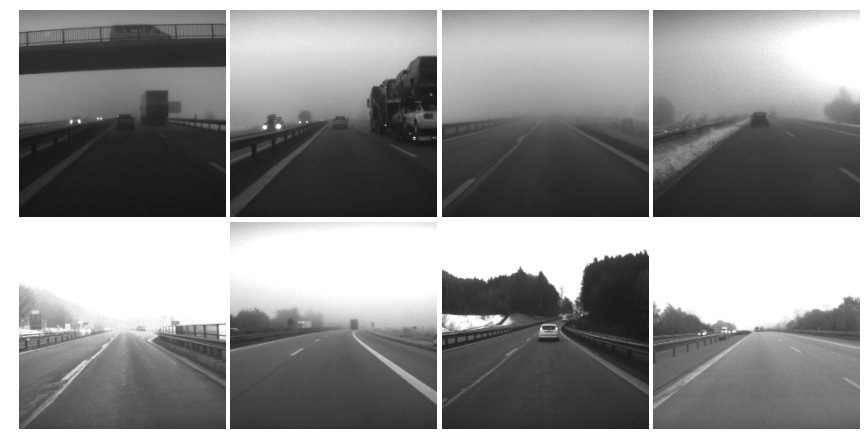

Fig. 7. Exemplary classification results. From top left to bottom right: The first two images show examples were falsely due to passing a bridge respectively overtaking a truck clear weather situations were detected (false negatives). The next two images show examples were our method has almost always detected fog situations correctly (true positives), this was e.g. the case for free road or for subsequent drives. The first two images of the second row show examples were fog was falsely detected (false positives). This occurred spontaneously and mostly in low-fog labeled images. The last two images show scenes correctly recognized as clear weather conditions (true negatives), one with basically high and one with basically low contrast, were both were trained with the same sample set.

From the recordings of night-time scenes we selected those in which was clearly the presence of fog respectively no presence of fog to see. To better assess the visibility, we periodically switched on the high beam during the measurement runs, if possible.

To evaluate the night-time scenes with high beam a 4-fold cross-validation was used. For this purpose four sample sets were created, each consisting of 1,800 images. Each sample set contains one half fog and the other half fog-free images. Thus 7,600 images were incorporated into the evaluation of night-time scenes with high beam. As could be seen in Tab. II all methods achieved very good results, wherein the Wavelet features and our method are slightly better than the Roser features with detection rates of $99.45 \%$ respectively $99.48 \%$.

\begin{tabular}{|c|c|c|c|c|}
\hline & & $\begin{array}{l}\text { Spectral } \\
\text { Features }\end{array}$ & $\begin{array}{c}\text { Roser } \\
\text { Features }\end{array}$ & Wavelets \\
\hline \multirow{3}{*}{ 氶 } & Acc & 0.9948 & 0.9841 & 0.9945 \\
\hline & $\mathrm{Tp}$ & 0.9940 & 0.9968 & 0.9932 \\
\hline & $\mathrm{Tn}$ & 0.9955 & 0.9714 & 0.9958 \\
\hline \multirow{3}{*}{$\begin{array}{l}\vec{\Delta} \\
\text { Dे } \\
\text { गे }\end{array}$} & Acc & 0.0048 & 0.0237 & 0.0031 \\
\hline & $\mathrm{Tp}$ & 0.0070 & 0.0048 & 0.0041 \\
\hline & $\mathrm{Tn}$ & 0.0088 & 0.0483 & 0.0070 \\
\hline \multirow{3}{*}{ 荡 } & Acc & 1.0000 & 0.9979 & 0.9984 \\
\hline & $\mathrm{Tp}$ & 1.0000 & 0.9968 & 0.9979 \\
\hline & $\mathrm{Tn}$ & 1.0000 & 0.9989 & 0.9989 \\
\hline \multirow{3}{*}{$\begin{array}{l}\overrightarrow{\tilde{a}} \\
\overrightarrow{0} \\
\overrightarrow{3}\end{array}$} & Acc & 0.9847 & 0.9232 & 0.9874 \\
\hline & $\mathrm{Tp}$ & 1.0000 & 0.9989 & 0.9958 \\
\hline & $\mathrm{Tn}$ & 0.9695 & 0.8474 & 0.9789 \\
\hline
\end{tabular}

TABLE II: Evaluation results for night-time scenes and high beam.

To evaluate night-time scenes with low beam an 8-fold cross-validation was used. For this purpose eight sample sets were created, each consisting of 4,250 images. Each sample set contains one half fog and the other half fog-free images. Thus 34,000 images were incorporated into the evaluation of night-time scenes with low beam. As could be seen in Tab. III in this case our method outperforms the others. Besides the highest recognition rate of $99.18 \%$ the lowest standard deviation of the single results of the cross-validation was achieved. Furthermore it achieves the best result for the worst single result with $97.65 \%$ accuracy.

\begin{tabular}{|c|c|c|c|c|}
\hline & & $\begin{array}{l}\text { Spectral } \\
\text { Features }\end{array}$ & $\begin{array}{l}\text { Roser } \\
\text { Features }\end{array}$ & Wavelets \\
\hline \multirow{3}{*}{ 苛 } & Acc & 0.9918 & 0.9683 & 0.9771 \\
\hline & $\mathrm{Tp}$ & 0.9888 & 0.9952 & 0.9911 \\
\hline & $\mathrm{Tn}$ & 0.9948 & 0.9414 & 0.9663 \\
\hline \multirow{3}{*}{$\begin{array}{l}\overrightarrow{0} \\
\overrightarrow{0} \\
\vec{n} \\
\vec{n}\end{array}$} & Acc & 0.0076 & 0.0673 & 0.0343 \\
\hline & $\mathrm{Tp}$ & 0.0111 & 0.0105 & 0.0109 \\
\hline & $\mathrm{Tn}$ & 0.0096 & 0.1328 & 0.0666 \\
\hline \multirow{3}{*}{ 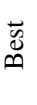 } & Acc & 1.0000 & 1.0000 & 1.0000 \\
\hline & $\mathrm{Tp}$ & 1.0000 & 1.0000 & 1.0000 \\
\hline & $\mathrm{Tn}$ & 1.0000 & 1.0000 & 1.0000 \\
\hline \multirow{3}{*}{$\begin{array}{l}\text { 范 } \\
\overrightarrow{0}\end{array}$} & Acc & 0.9765 & 0.7024 & 0.8736 \\
\hline & $\mathrm{Tp}$ & 0.9991 & 0.9986 & 0.9906 \\
\hline & $\mathrm{Tn}$ & 0.9539 & 0.4061 & 0.7567 \\
\hline
\end{tabular}

TABLE III: Evaluation results for night-time scenes and low beam.

In Fig. 8 some classification results are shown from the evaluation of night-time scenes with low beam. The first two 
images top left show two exemplary situations in which the presence of fog was correctly detected, one with free road and one with a vehicle ahead and rear fog lamp switched on. The following two images show two false classifications, in which no fog was mistakenly detected. Once by an oncoming vehicle and once by a vehicle driving ahead to high contrasts were generated in the image. Mostly, however, false positives were generated due to an erroneous imaging of the sensor, whereby horizontal gradients were generated in dark image regions. This phenomenon was evident only in the pre-filtered images, and is not shown here. The first two images in the second row show examples, where fog was mistakenly classified. In the first image there is light fog and the headlights of an oncoming vehicle produce weak edges to an already basically low contrast scene. In the second image produces an oncoming vehicle, which runs straight out of the image, a diffuse light which extends over large parts of the left side. The last two pictures show finally two scenes that were correctly classified as free of fog, one with free road in a low-contrast environment and another with dazzling by an oncoming, distant vehicle.
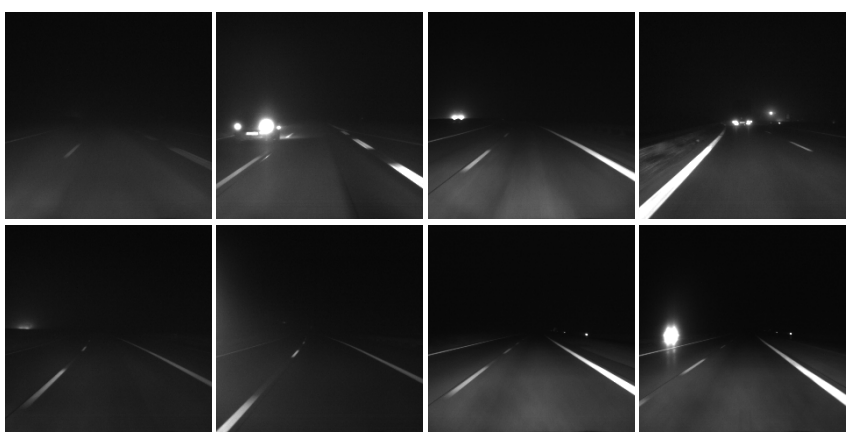

Fig. 8. Exemplary classification results for night-time scenes. From top left to bottom right: Each two examples of correctly detected fog scenes, falsely detected as fog-free scenes, falsely detected as fog scenes and correctly detected as fog-free scenes.

\section{CONCLUSION}

Frequency information of spectral features are well suited to distinguish between driving scenes with clear and foggy weather conditions. Compared to the features proposed by Roser and Moosmann and to the Wavelet features proposed by Serrano et al. with these the best detection rates were achieved in both day and night driving scenes. In daytime scenes, the result of an 8-fold cross-validation based on 44,000 images provided a detection rate of $95.35 \%$. The examination of night driving scenes were divided into images with high beam and with low beam switched. When high beam switched, a 4-fold cross-validation based on 34,000 images provided a detection rate of $99.48 \%$, while the other two methods, however, achieved similar good results. When low beam switched, an 8-fold cross-validation based on 34,000 images provided a detection rate of $99.18 \%$.

\section{ACKNOWLEDGMENT}

The authors would especially like to thank Martin Roser for supporting us with an implementation of his algorithm.

\section{REFERENCES}

[1] American Meteorology Glossary. (2012) Glossary of meteorology. [Online]. Available: http://amsglossary.allenpress.com/glossary

[2] Commission of Illumination, "International Lighting Vocabulary, CIE 17.4," 1987.

[3] M. Pavlić, H. Belzner, G. Rigoll, and S. Ilić, "Image based fog detection in vehicles," in IEEE Intelligent Vehicles Symposium, 2012, pp. 1132-1137.

[4] C. Busch and E. Debes, "Wavelet transform for analyzing fog visibility," IEEE Intelligent Systems, vol. 13, no. 6, pp. 66-71, 1998.

[5] D. Pomerleau, "Visibility estimation from a moving vehicle using the ralph vision system," in IEEE Conference on Intelligent Transportation Systems, 1997, pp. 906-911.

[6] N. Hautière, J.-P. Tarel, J. Lavenant, and D. Aubert, "Automatic fog detection and estimation of visibility distance through use of an onboard camera," Machine Vision and Applications, vol. 17, no. 1, pp. 8-20, 2006.

[7] N. Hautière, R. Labayrade, and D. Aubert, "Real-time disparity contrast combination for onboard estimation of the visibility distance," IEEE Transactions on Intelligent Transportation Systems, vol. 7, no. 2, pp. 201-212, 2006.

[8] N. Hautière, R. Labayrade, C. Boussard, J.-P. Tarel, and D. Aubert, "Perception through scattering media for autonomous vehicles," in Autonomous Robots Research Advances. Nova Science Publishers, Inc., 2008, ch. 8, pp. 223-267.

[9] S. Bronte, L. M. Bergasa, and P. F. Alcantarilla, "Fog detection system based on computer vision techniques," IEEE Intelligent Transportation Systems, vol. 12, pp. 1-6, 2009.

[10] R. Gallen, A. Cord, N. Hautière, and D. Aubert, "Towards night fog detection through use of in-vehicle multipurpose cameras," in Intelligent Vehicles Symposium, 2011, pp. 399-404.

[11] M. Szummer and R. W. Picard, "Indoor-outdoor image classification," in Proceedings of IEEE International Workshop on Content-Based Access of Image and Video Database, 1998, pp. 42-51.

[12] A. Guérin-Dugué and A. Oliva, "Classification of scene photographs from local orientations features," Pattern Recognition Letters, vol. 21, no. 13-14, pp. 1135-1140, 2000.

[13] A. Savakis and J. Luo, "Indoor vs. outdoor classification of consumer photographs," in Proceedings of IEEE International Conference on Image Processing, vol. 2, 2001, pp. 745-748.

[14] N. Serrano, A. Savakis, and J. Luo, "A computationally efficient approach to indoor/outdoor scene classification," in Proceedings of the 16th International Conference on Pattern Recognition, vol. 4, 2002, pp. $146-149$.

[15] A. Quattoni and A. Torralba, "Recognizing indoor scenes," in IEEE Conference on Computer Vision and Pattern Recognition., 2009, pp. 413-420.

[16] A. Vailaya, A. Jain, and H. J. Zhang, "On image classification: City images vs. landscapes," Pattern Recognition, vol. 31, no. 12, pp. 19211935, 1998.

[17] A. Vailaya, A. Jain, M. Figueiredo, and H. Zhang, "Content-based hierarchical classification of vacation images," in Proceedings of the IEEE International Conference on Multimedia Computing and Systems, vol. 2, 1999, pp. 518-523.

[18] A. Oliva and A. Torralba, "Modeling the shape of the scene: A holistic representation of the spatial envelope," International Journal of Computer Vision, vol. 42, no. 3, pp. 145-175, 2001.

[19] M. Boutell, A. Choudhury, J. Luo, and C. Brown, "Using semantic features for scene classification: how good do they need to be?" in IEEE International Conference on Multimedia and Expo, 2006, pp. 785-788.

[20] R. Datta, D. Joshi, J. Li, and J. Z. Wang, "Image retrieval: Ideas, influences, and trends of the new age," ACM Computing Surveys, vol. 40, no. 2, pp. 1-60, 2008.

[21] M. Roser and F. Moosmann, "Classification of weather situations on single color images," in IEEE Intelligent Vehicles Symposium, 2008, pp. $798-803$.

[22] R. Kimmel, M. Elad, D. Shaked, R. Keshet, and I. Sobel, "A variational framework for retinex," International Journal of Computer Vision, vol. 52, no. 1, pp. 7-23, 2003.

[23] E. H. Land, "The Retinex Theory of Color Vision," Scientific American, vol. 237, no. 6, pp. 108-128, 1977.

[24] B. Manjunath and W. Ma, "Texture features for browsing and retrieval of image data," IEEE Trans. PAMI, vol. 18, pp. 837-842, 1996. 